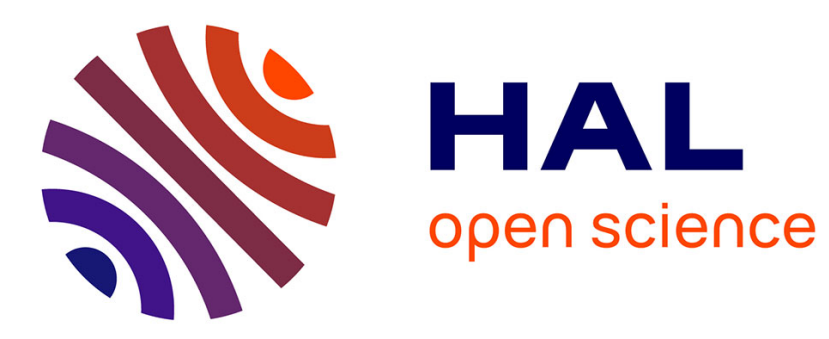

\title{
Hétérogénéité, cohésion et cohérence : le statut de l'anecdote digressive dans un récit de voyage
}

\author{
Véronique Magri-Mourgues
}

\section{To cite this version:}

Véronique Magri-Mourgues. Hétérogénéité, cohésion et cohérence: le statut de l'anecdote digressive dans un récit de voyage. D. Denis, A. Jaubert. Cohésion et cohérence, études de linguistique textuelle, ENS éditions, pp.134-148, 2005. hal-01225997

\section{HAL Id: hal-01225997 \\ https://hal.science/hal-01225997}

Submitted on 10 Nov 2015

HAL is a multi-disciplinary open access archive for the deposit and dissemination of scientific research documents, whether they are published or not. The documents may come from teaching and research institutions in France or abroad, or from public or private research centers.
L'archive ouverte pluridisciplinaire HAL, est destinée au dépôt et à la diffusion de documents scientifiques de niveau recherche, publiés ou non, émanant des établissements d'enseignement et de recherche français ou étrangers, des laboratoires publics ou privés. 


\title{
HÉTÉROGÉNÉITE, COHÉSION ET COHÉRENCE : LE STATUT DE L'ANECDOTE DIGRESSIVE DANS UN RÉCIT DE VOYAGE
}

\author{
Véronique Magri-Mourgues \\ Université Nice Sophia-Antipolis \\ UMR 7320 Bases, Corpus, Langage
}

Selon le Dictionnaire de poétique et de rhétorique de Henri Morier, l'anecdote est un exemple de digression, partie du discours où l'auteur s'éloigne du sujet, au sens strict de l'étymon. L'anecdote implique a priori une dimension narrative qui la distingue d'autres types de digressions comme la séquence descriptive ${ }^{1}$ ou encore l'inventaire savant ou note savante $^{2}$; elle a ce statut paradoxal de l'élément « en marge de ». Elle est comme décalée par rapport au discours qui l'englobe cependant et lui donne sa raison d'être. Dans le récit de voyage, elle accède à un statut particulier puisque sa présence, inversement, fonde le discours qui l'inclut, devenant un principe générique: tout récit de voyage peut être envisagé comme collection d'anecdotes.

Toutefois, empruntant aux techniques du récit enchâssé, l'anecdote est un motif de discontinuité et d'hétérogénéité évident : elle induit une collision entre deux types de récits et provoque une rupture de la cohésion textuelle du récit-cadre.

Comme elle relève d'une esthétique du fragment, elle se propose comme un texte achevé avec sa propre cohésion / cohérence à l'intérieur d'un macrotexte qu'elle contribue à ouvrir en permanence, rompant la linéarité discursive par des détours répétés. La frontière entre le texte-cadre et le texte enchâssé est alors mise en scène par le narrateur qui, tout en exhibant l'hétérogénéité, tend à lisser les disparités formelles pour retrouver à chaque fois le fil du discours, pour rendre sa cohésion au texte et en assurer la lisibilité. La chaîne des anecdotes qui se constitue comme un paradigme et qui est perçue à la lecture, donc du côté de la réception, peut encore servir la cohésion textuelle jusqu'à prendre le pas sur le récit-cadre : et si, d'une anecdote à l'autre, se construisait comme un discours second, parallèle au discours cadre, et pourtant primordial puisque porteur de la plus forte valeur référentielle, gage de l'authenticité revendiquée par le récit de voyage ? N'est-on pas confronté à une aporie ? Postuler l'existence de digressions présuppose la cohérence de l'œuvre orientée par un fil directeur et perturbé ponctuellement par des excursus. Paradoxalement, l'anecdote dite digressive ne serait-elle pas en fait le principe de

\footnotetext{
${ }^{1}$ Qu'on pourrait illustrer entre autres par la description d'un rachitique, p. 40.

2 Voir la note savante sur le sycomore d'Égypte, « digression de botanique », p. 24.
} 
cohérence essentiel du récit de voyage ? L'étude porte sur Maxime Du Camp, Le Nil, Egypte et Nubie, [1854], Paris, Hachette, 1877.

\section{L'anecdote comme élément hétérogène}

J'ai constitué deux sous-corpus, l'un regroupant l'ensemble des anecdotes digressives du $\mathrm{Nil}$, l'autre le reste du texte, par conséquent exclusif des anecdotes, que j'appellerai le récit-cadre. Je m'intéresse aux anecdotes que je nomme digressives par opposition à celles qui mettent en scène le voyageur même et qui sont contemporaines du voyage. Si on considère que la digression est un «endroit de l'ouvrage où l'on traite de choses qui paraissent hors du sujet principal $»^{3}$, l'anecdote centrée autour du voyageur est à exclure du corpus d'étude. Un autre procédé d'écriture, propre au récit de voyage, est alors en jeu : l'hérö̈sation du voyageur qui passe par la multiplication des anecdotes où il tient le rôle principal. Ces anecdotes sont fondues dans le discours du voyageur et ne présentent pas les mêmes caractéristiques que celles où le narrateur - voyageur est hétérodiégétique et qui sont antérieures au voyage et à la narration. Ce type d'anecdote se constitue comme une unité que des marqueurs explicites intégrés dans le récit-cadre isolent et désignent. Il peut s'agir de marques typographiques comme les deux-points qui annoncent l'anecdote ou encore de guillemets en ouverture et en clôture qui délimitent sans ambiguïté l'anecdote prise en charge par un tiers. En amont de l'anecdote, des termes métatextuels peuvent aussi la désigner clairement comme « histoire » autonome, qui sont relayés ensuite par une variation de temporalité voire une variation complète et temporaire de la scénographie 4 ou même repris en écho pour clore l'anecdote. Dans ce corpus, la séquence digressive se distingue clairement du récit-cadre comme un fragment clos, comme une halte sur le parcours du voyageur ; toute confusion éventuelle entre les différents plans est ainsi évitée.

En dépit du déséquilibre des deux ensembles ainsi établis qui nuit à l'exploitation complète des résultats, le vocabulaire spécifique 5 de chacun des deux fournit une première approche par la mise en évidence d'indices grammaticaux différentiels : cette comparaison fournit deux séries de résultats si on prend en compte deux points sensibles

\footnotetext{
3 B. Dupriez 1984, p. 157.

4 La scénographie peut être définie comme le « foyer de coordonnées qui sert de repère, directement ou non, à l'énonciation : les protagonistes de l'interaction langagière, énonciateur et coénonciateur, ainsi que leur ancrage spatial et temporel. » (Maingueneau 1993, p. 121).

5 Les deux ensembles ont été comparés statistiquement par le biais du logiciel Hyperbase mis au point par Étienne Brunet. J'ai pu ainsi établir la liste du vocabulaire spécifique de chacun des deux ensembles l'un par rapport à l'autre. Ces résultats fournissent une première esquisse des caractéristiques lexicales différentielles, grâce au calcul automatique des écarts réduits.
} 
du discours, le verbe et les marques personnelles, pronoms ou adjectifs. La comparaison de ces catégories grammaticales met en évidence une rupture de la cohésion, sur deux plans, temporel et énonciatif.

\subsection{La discontinuité temporelle}

Toutes les formes verbales spécifiques de l'ensemble constitué par les anecdotes sont des formes de passés simples ou d'imparfaits («avait, était, fit, répondit, arrêta, furent ») tandis que le récit-cadre est caractérisé par une forme verbale au présent « est ».

La rupture de la cohésion textuelle est évidente et transparaît au travers de marques linguistiques claires. Le discours du narrateur - voyageur est orienté linéairement puisqu'il suit les étapes du voyage réel, déterminées elles-mêmes par la contiguiité spatiale des lieux traversés. L'insertion des anecdotes, qui relèvent du principe de la narration ultérieure, est à l'origine de la perturbation du déroulement chronologique Dans le cas de l'anecdote digressive, la narration quitte temporairement l'histoire principale pour «flirter » avec d'autres micro - histoires. Différents cas se présentent, selon qu'on a affaire à des anecdotes historiques ou légendaires. Les anecdotes historiques sont datées tandis que les anecdotes légendaires sont situées dans un hors-temps indéfini dénoté par des formules comme « il y a bien longtemps »:

Une fois, il y a bien longtemps, un cheikh de derviches qu'on nommait Hadji Abdoul Aziz marchait péniblement sous le soleil et dans la poussière de ce sentier difficile. (p. 303)

Il y a bien longtemps, sous le règne d'un sultan mameluk, un santon très-pieux et faiseur de miracles, nommé Saad-Eddin (bonheur de la religion), dressa ses tentes près du Kaire, avant de traverser les sables et la mer rouge pour se rendre à la Mecque. (p. 60)

ou encore comme l'adverbe « autrefois » dont on compte deux occurrences insérées dans les anecdotes :

Un saint homme vivait autrefois au milieu des ruines d'Antinoë. (p. 107)

Autrefois il y avait à Paris, au jardin des plantes, un palmier femelle. (p. 183)

sans parler de l'expression conventionnelle « il était une fois » :

Il était une fois un pharaon d'Égypte, très sage, très puissant et très riche. Il habitait à Memphis de magnifiques palais, sur les bords du Nil. (p. 70) 
inaugurant une anecdote qui rappelle explicitement le conte de Cendrillon.

Outre la question de l'insertion de la séquence anecdotique dans la trame narrative et de la brisure qu'elle introduit dans cette linéarité, la temporalité interne de l'anecdote est un autre motif de discontinuité temporelle. Associés à la forme verbale « est », les articles pluriel « des » et «les » caractérisent le récit-cadre. L'anecdote isole un instant unique : le syntagme «un jour » intervient à trois reprises, relayé par « une fois » dont trois occurrences sur quatre se situent dans des anecdotes: la seule occurrence qui fait exception appartient à une locution adverbiale distributive ${ }^{6}$.

Un jour qu'il se promenait sous les galeries de la mosquée de la Mecque, il pensa à Amr et regarda du côté du Kaire. (p. 49)

Un jour je partis... (p. 203)

Un jour qu'il était là, selon sa coutume, vêtu de sa belle robe blanche et disant des maximes dont chacun s'émerveillait, un aigle passa au-dessus de sa tête, ouvrit les serres et en laissa échapper quelque chose de brillant qui vint tomber sur ses genoux. (p. 70)

Ces observations confirment l'idée que le récit de voyage vise la généralisation, le stéréotype, tandis que l'anecdote s'organise autour d'un événement unique et particulier. Cette particularité temporelle permet de distinguer l'anecdote digressive de séquences typiques, que l'on pourrait appeler des scènes ; même si elles s'appuient sur un spectacle contemporain du voyage initial, celles-ci suivent une démarche inductive qui vise la généralisation et le stéréotype, l'image d'Orient : c'est le cas par exemple des scènes du mariage ou de la danse orientale auxquelles assiste le voyageur. Les scènes sont données comme uniques mais représentatives. Elles se chargent d'une valeur omnitemporelle dont est dépourvue l'anecdote digressive ${ }^{7}$.

\subsection{L'hétérogénéité énonciative}

L'ensemble des anecdotes se caractérise par la prépondérance des marques de la troisième personne et de la deuxième personne du singulier, pronoms personnels « il », « lui » et « tu » et adjectif possessif « sa » et «ton». Le Trésor de la Langue française envisage le système des personnes comme un point stratégique du discours et établit une

\footnotetext{
6 «Lorsqu'on y a goûté une fois, on ne peut s'en désaccoutumer », p. 313.

7 Voir par exemple la scène du dossêh (piétinement), p. 60 sq.
} 
classification générique ${ }^{8}$ des œuvres selon l'emploi des personnes. Le pronom « il » est cependant ambigu puisqu'il peut jouer le rôle d'un simple indice grammatical. En analysant d'un peu plus près les contextes du pronom «il », on relève en fait une minorité d'exemples où « il » est utilisé dans une tournure impersonnelle du type «il faut », « il y a » ou « il y avait », ou encore « il me plaît » et «il est resté ». Les autres occurrences de « il » illustrent des cas de pronom personnel de troisième personne, véritable substitut d'un groupe nominal et notamment comme sujet d'un verbe de parole ; ce qui confirme par conséquent que l'accent est mis, pour l'anecdote, sur la transmission supposée orale des histoires. La primauté de la troisième personne signalerait un dispositif narratif tandis que celle de la seconde personne dénoterait une structure dialogique. Ce dernier fait est d'autant plus intéressant que pour le corpus générique « récit de voyage » de la base de données Frantext, le pronom «tu » est largement déficitaire ${ }^{9}$, ce qui contraste avec le corpus des anecdotes. On constate également la primauté du pronom «vous» dans l'ensemble constitué par les anecdotes. L'émergence de la personne de l'allocution paraît bien un signe distinctif de l'anecdote digressive, qui se trouve mise en voix dans Le Nil. Le décrochage énonciatif qui est ainsi impliqué est un motif d'hétérogénéité entre le récitcadre et l'anecdote.

\section{Mise en voix des anecdotes}

Les anecdotes de ce récit de voyage adoptent une structure de dialogue, qu'elles se construisent entièrement sur un dialogue ou bien qu'elles insèrent des bribes de discours direct. Le récit-cadre est pris en charge par un narrateur unique qui peut déléguer la parole à une tierce personne pour donner voix à une anecdote. Le relais narratif fait entendre la parole de l'Autre. Diverses réalisations de la parole rapportée sont illustrées et définissent une distance énonciative variable entre le discours porteur et le récit rapporté ; on relève un exemple de discours narrativisé dans l'anecdote - sommaire :

Le cheikh commença alors une longue histoire, en jurant qu'il disait la vérité, et nous parla d'hyènes et de chacals qui attaquaient ses moutons. (p. 298).

Le dialogue peut constituer une anecdote tout entière comme l'histoire autobiographique du drogman Joseph Brichetti, racontée au voyageur-narrateur, institué auditeur. Des bribes de dialogue sont insérées dans la plupart des anecdotes jusqu'à cette invective du

\footnotetext{
8 Chacune des œuvres du corpus du Trésor de la Langue française a été classée dans l'un des trois ensembles, prose, vers, poème en prose, puis dans l'un des trois sous-ensembles, le soliloque lorsque les marques de la première personne dominent, le dialogue lorsque ce sont celles de la seconde personne qui sont le plus représentées, par exemple.

${ }^{9}$ Voir V. Magri 1995, p. 324.
} 
khalife Omar à un pilier récalcitrant à ses ordres et qui lui répond dans une prosopopée merveilleuse :

Il se tourna alors vers un des piliers qui l'entouraient et lui ordonna de se rendre au Kaire sans délai et de prendre la place de la colonne défectueuse. Le pilier trembla légèrement et reprit son immobilité.

Omar, étonné, le poussa violemment avec la paume de sa main en réitérant son ordre ; le pilier s'agita comme pris de vertige, tourna sur lui-même, mais n'obéit pas ; Omar, furieux, le frappa de son courbach, en s'écriant cette fois : «au nom de Dieu clément et miséricordieux, va ! - Pourquoi avais-tu oublié d'invoquer Dieu ? » répondit le pilier en prenant son vol et en venant tout à coup se placer naturellement devant le kébla de la mosquée égyptienne. (p. 50)

Les propos rapportés sont quelquefois seulement signalés par des incises attendues telles « d'après les gens du pays »:

D'après les gens du pays, tous les soirs à minuit on aperçoit venir de grands fantômes blancs qui s'appuient sur des lances d'or, et dont le front est armé de cornes d'ivoire; leur longue barbe flotte sur la poitrine ; leurs yeux sont si perçants qu'ils voient au delà du ciel empyrée ; ils marchent lentement, deux par deux, chacun suivi par un chien qui a un oiseau sur la queue et un scarabée d'argent sur le dos ; ils font processionnellement le tour des rochers en remuant la tête comme des idoles chinoises.

Quand ils ont terminé cette promenade circulaire, un corbeau vient, qui leur dit : « vous pouvez vous asseoir »; puis il s'envole à tire-d'aile. (p. 165)

ou bien par la formule suivante, volontairement désinvolte, qui décharge le narrateur de toute responsabilité :

Cette légende est difficile à raconter, cher ami, néanmoins je veux te la dire ; si elle est trop excentrique, la faute en est aux Arabes et non pas à moi. (p. 184)

Une seule anecdote est exclusivement narrative, celle qui rappelle le conte de Cendrillon - par conséquent le changement de régime énonciatif qui caractérise habituellement les anecdotes est ici absent - mais d'autres indices la signalent comme un fragment autonome et peut-être « détachable », notamment une mise en récit particulière. 
Le calcul de l'indice pronominal, élaboré et mis en œuvre par Charles Muller, permet une autre comparaison entre les deux corpus : Charles Muller prend en compte les pronoms personnels non ambigus qu'il appelle les pronoms de dialogue, « je, me, moi, tu, te, toi, nous, vous » ainsi que leurs formes élidées « j', m', t' » soit onze formes et les adjectifs et les pronoms possessifs correspondants, «mon, le mien, ton, le tien, notre, le nôtre, votre, le vôtre » et leur féminin et leur pluriel respectifs, le cas échéant, soit vingt-deux formes. Il calcule le rapport entre ces deux séries de formes dans un corpus de textes classiques et constate que l'indice varie en fonction du genre du texte, atteignant les valeurs les plus hautes dans le langage parlé (le discours oral privilégie l'emploi du personnel contre le possessif lorsque la langue offre la possibilité de choix) ; ce quotient fonctionne comme un indice stylistique. Pour le récit de Du Camp, le calcul de l'indice pronominal donne un résultat égal à 4,3 pour le corpus qui exclut les anecdotes et égal à 3,6 pour l'ensemble des anecdotes qui, par conséquent aurait une tendance littéraire légèrement plus marquée. Toutefois, l'écart entre les deux rapports est trop faible pour qu'on puisse en tirer une conclusion sûre et convaincante.

Le genre du récit de voyage est dit référentiel puisqu'il prétend refléter une vision du réel. Les anecdotes, elles, affichent un parti pris fictionnel quand on a affaire à une histoire merveilleuse, une légende. C'est là un autre motif de rupture de la cohésion, qui se place dans le domaine générique. Il est admis que le récit de voyage est un genre à la croisée d'autres genres comme l'autobiographie, l'ouvrage scientifique, le roman. L'insertion de l'anecdote manifeste la traversée du texte qui se revendique comme fictionnel et qui s'accompagne de la suspension de la valeur de vérité accordée à l'histoire et de la suspension de la crédulité du lecteur. Le Nil fournit le parangon du texte fictionnel en l'espèce du conte qui rappelle celui de Cendrillon. Placé après l'anecdote, un parallèle cocasse entre la légende et le point de vue savant étayé par une débauche de noms propres accentue le contraste générique :

Je t'en prie, cher Théophile, ne parle de la légende que je viens de te raconter à aucun membre de l'Académie des Inscriptions et Belles-Lettres ; tout savant te dirait immédiatement, en levant les épaules, qu'il ne faut pas ajouter foi à de semblables sornettes, bonnes à endormir les enfants, et que la troisième pyramide a été bâtie par Mycérinus, nommé par les Égyptiens Menkéré ou Menkarès.

Il compulserait les textes et te prouverait que Rhodopis, originaire de Thrace, fut esclave d'Idamon, fils d'Éphestopolis le samien, et compagne d'esclavage d'Ésope le fabuliste ; qu'amenée en Égypte par Xantus pour faire le métier de courtisane, elle fut rachetée à prix d'or par le frère de Sapho, Charamis De Mytilène, fils de Scamandronyme (...) 
À cela je n'aurais rien à répondre, sinon, que je trouve la légende jolie, et qu'il me plaît d'y croire, comme je crois à Cendrillon. (p. 72-73)

Trouver des marqueurs linguistiques sûrs du partage générique entre genre référentiel et genre fictionnel est cependant peine perdue si on excepte le cas d'indices liminaires métatextuels précisant l'enjeu du texte ou bien du type «il était une fois ». On donne quelquefois la présence de noms propres authentiques ou de gloses savantes comme indices de référentialité. Mais il serait aisé de montrer que là encore, le genre qui se revendique comme fictionnel mime de fait ces facteurs de vraisemblance. Plus intéressant est justement le brouillage des genres à l'œuvre chez Du Camp.

L'emploi de l'appareil argumentatif au service d'une explication non rationnelle est un des procédés qui assurent ce brouillage. La mise en évidence d'outils de cohésion textuelle pour une anecdote merveilleuse fonctionne comme support de l'ironie :

Voici l'heure où les fantômes blancs vont apparaître. Certes, si jamais ruines ont été visitées par les esprits, ce sont bien celles-là ! Où trouveraient-elles donc, ces pauvres âmes errantes qui flottent dans les espaces, un ciel plus étoilé, de plus larges colonnades, des échos plus sonores, un silence plus profond, des images plus belles et un plus religieux recueillement? Je comprends que les Arabes, toujours cherchant le merveilleux, aient peuplé ces solitudes avec les génies dont Éblis est le chef et que Soliman Ben Daoud avait enchaînés sous son trône. (p. 228-229)

Après le récit d'une anecdote qui met en scène un anachorète dont l'âne, fidèle serviteur, a été dévoré par un crocodile et qui, désespéré et furieux, lance un anathème contre les crocodiles, le narrateur feint de conclure sérieusement :

La malédiction a été féconde, car, depuis, jamais un crocodile n'a pu franchir le Nil au-delà de Cheikh-Abadeh. (p. 108)

Dans l'exemple suivant, ce n'est que la dernière phrase du paragraphe qui permet d'interpréter, à rebours, les phrases précédentes comme du discours indirect libre, bivocal par définition, et permettant, par conséquent, la superposition des niveaux énonciatifs :

Des mouettes en grand nombre voltigeaient autour de ma cange et se précipitaient avec des cris aigus sur le pain que les matelots leur jetaient.

Tu crois peut-être qu'elles le mangent ! Non, elles vont le déposer sur un banc placé devant le tombeau de Cheikh-Saïd, afin qu'il puisse servir de nourriture aux pèlerins et aux voyageurs. 
Voilà du moins ce que me raconta Reïs-Ibrahim. (p. 107-108)

L'explication rationnelle (les mouettes mangent les miettes de pain) est rejetée ironiquement comme absurde, tandis que l'explication merveilleuse est assertée sans aucune mise à distance visible. Le caractère intentionnel de l'action des mouettes est, au contraire, renforcé par la locution conjonctive finale, «afin que » et par la périphrase modale "puisse servir ». La source énonciative révélée à la fin du paragraphe oblige à une relecture de l'extrait et à une interprétation humoristique. Quelques pages plus loin, Du Camp poursuit en mêlant décor réel et conte merveilleux :

Et j'ai pensé que c'était là que devaient habiter les héros des contes de l'Orient, la fée des roseaux verts et le génie des sombres eaux. (p. 137)

Ce brouillage générique joue en faveur du rétablissement de la cohésion entre anecdotes merveilleuses et discours du voyageur. Des procédés linguistiques assurent de même le « rattrapage » temporel et énonciatif et ont pour vocation de lisser l'hétérogénéité tout en l'exhibant.

\section{L'hétérogénéité montrée}

2.1. Mise en scène de la limite : les marqueurs d'intégration

L'hétérogénéité est montrée au bénéfice de la continuité discursive. Même si la séquence digressive est clairement délimitée dans ce corpus, le souci du narrateur est toujours de prévenir l'objection du hors-sujet et, tout en exhibant les limites de l'îlot descriptif, de l'intégrer dans la trame discursive en essayant de le légitimer. Le vocabulaire métatextuel désigne clairement la séquence digressive comme une histoire close avec des termes comme « anecdote » même ou ses avatars tels « histoire », « historiette », « légende ».

Les éléments se répondent quelquefois symétriquement de part et d'autre de la séquence anecdotique, à la manière de parenthèses ouvrante et fermante, par exemple avec l'emploi de « voici » utilisé selon la distribution canonique en ouverture de l'anecdote; la clôture étant assurée par l'anaphore lexicale («cet événement merveilleux ») : 
C'est en commémoration de cet événement merveilleux qu'on exécute le dossêh ; seulement, à cette heure, au lieu d'étendre des bouteilles et des flacons sous les pas du cheval, on y met des hommes. (p. 60)

ou bien, par la reprise lexicale d'un même verbe qui subit une variation aspectuelle et un changement de sujet. La place inversée de celui-ci établit par ailleurs une structure en chiasme qui paraît bien fonctionner comme un indice de clôture :

Les Arabes appellent cette dernière pyramide le monument de la fille. Est-ce qu'ils ont eu connaissance de cette légende charmante que racontent Hérodote, Diodore et Strabon ? (p. 70)

Je t'en prie, cher Théophile, ne parle de la légende que je viens de te raconter à aucun membre de l'Académie des Inscriptions et Belles - Lettres. (p. 72)

Une seule anecdote est dépourvue d'indices métatextuels de clôture ou plutôt la fin de l'histoire racontée coïncide avec la fin de sa narration. Il s'agit de l'anecdote historique du massacre des mameluks en 1811 ordonné par Méhémet-Ali qui se termine par l'exécution d'un rescapé : la dernière phrase, avant la reprise de la narration du voyage, est consacrée à l'exécution du seul homme qui a échappé au massacre : « Huit jours après, il fut saisi et décapité » (p. 79) C'est l'exemple le plus parfait de lissage entre les deux types de récits. Quoi qu'il en soit, Du Camp en bon narrateur, cultive l'art des transitions.

\section{L'anaphore}

L'insertion d'une anecdote est motivée par la logique associative : le caractère fortuit de l'anecdote s'inscrit comme naturellement dans les aléas d'une conversation ou dans ceux de l'itinéraire spatial. Le cheminement discursif est similaire au déplacement dans l'espace qui fait rencontrer divers lieux sur lesquels rebondit l'imagination du voyageur : celui-ci évoque des anecdotes qui se sont déroulées dans les lieux mêmes qu'il traverse mais à une autre époque ; l'adverbe de lieu déictique et anaphorique « là », en maintenant l'identité de la référence, sert alors de relais entre le discours-cadre et l'anecdote enchâssée.

On arrive au vieux Kaire, la Babylone de l'ancienne Égypte.

Ce fut là qu'Amr-Ben-El-Âs planta sa tente sur laquelle un pigeon s'arrêta et ce fut là qu'il bâtit sa mosquée. (p. 47) 
C'est là que les mameluks furent massacrés sans possibilité de fuite ou de défense, le 1er mars 1811, par ordre de Méhémet - Ali. (p. 79)

La variation d'un seul paramètre ouvre un feuilleté temporel qui se déplie autour du lieu ; celui-ci joue alors le rôle de pivot explicite et assure l'enchaînement des deux types de discours.

Le fil du discours est aussi maintenu lorsqu'un lien logique de cause à effet est apparemment invoqué :

Puisque je te parle de Méhémet-Ali, [...] je te raconterai sur lui une courte anecdote fort caractéristique et peu connue. (p. 9)

Mais cette proposition subordonnée qui table sur l'évidence du « puisque » inaugural comme connecteur entre les deux récits, ne fait que jouer sur les hasards du style épistolaire, qui veut mimer le naturel d'une conversation ordinaire ${ }^{10}$. Cette phrase est une charnière, à la fois rattachée en amont dans le texte et en aval, par le biais du futur équivalent ici à un futur périphrastique de valeur aspectuelle.

\section{Le lien de causalité}

Une anecdote se donne comme explication d'une observation visuelle première :

Près du kébla principal de la mosquée, parmi les colonnes qui l'entourent, il en est une dont le fût se distingue par une dépression assez sensible et par une veine qui semble avoir été cinglée après coup. (p. 49)

«Semble » sert de tremplin à l'explication légendaire. Là encore, la cohésion est maintenue par le biais du lien logique de causalité ; le verbe modalisateur « semble » joue un double rôle : il est à la fois l'élément qui autorise et même réclame une interrogation et une réponse à cette interrogation, fournie par l'anecdote, mais aussi l'élément sur lequel le discours pivote et bascule dans l'imaginaire et le légendaire.

\section{Rattrapage temporel}

Pour les anecdotes qui introduisent un décalage dans le temps, la clôture de la séquence assure le « rattrapage temporel » par le biais des adverbes «encore » ou « depuis », qui établissent la continuité du passé à l'époque de la narration. On glisse d'un plan d'énonciation à l'autre; il se produit une interaction entre deux époques, le moment de

10 Voir A. Jaubert, 1993. 
l'histoire et le moment de l'énonciation. L'emploi de ces adverbes temporels indifféremment pour les anecdotes historiques ou légendaires contribue à brouiller les catégories.

La dépression et la veine cinglée qu'on y voit encore sont la trace de la main et du coup de fouet du khalife. (p. 50)

On montre encore, sur une pointe de rochers placée près du Nil, un marabout qui est le tombeau de Bellal. (p. 172)

« Garde tes fruits, homme méchant, répondit Hadji Abdoul-Aziz ; qu'ils deviennent aussi durs que ton cœur, et que ton champ soit aussi stérile que ton âme. » Aussitôt les pastèques furent changées en blocs de granit, et le sable envahit le champ, qui depuis ne put jamais rien produire. (p. 304)

\section{Rattrapage énonciatif}

Un « rattrapage » peut gommer les disparités entre deux niveaux énonciatifs d'une part en jouant sur les modalités de la parole rapportée, d'autre part en quittant ostensiblement le domaine de l'anecdotique pour retrouver la sphère de l'énonciation première et s'élever, dans une démarche inductive, au cas général. L'exemple suivant illustre le premier type de lissage énonciatif :

Mon guide, Temsah, me raconta à voix basse que toutes les nuits on voyait nager, sur les eaux fangeuses et épaisses de ce lac, un vaisseau d'or manœuvré par des femmes d'argent et trainé par un gros poisson bleu. Plusieurs Arabes ont déjà tenté de s'en emparer, mais le navire disparaît en fumée dès qu'on s'en approche. (p. 232)

Cet extrait commence par une phrase au style indirect : le verbe de parole introducteur «me raconta» identifie clairement la source énonciative. A mesure que la phrase se déplie, l'identité du locuteur premier s'estompe pour disparaître complètement dans la seconde phrase. L'extrait se termine en effet sur un segment dépourvu de toute marque modalisatrice et qu'on pourrait attribuer à la voix même du narrateur. En contexte cependant, la phrase peut être interprétée comme un fragment de discours indirect libre, illustration parfaite de la bivocalité du discours. Il en résulte une homogénéisation des deux discours, celui de Temsah, celui du voyageur-narrateur. 
Le second type de lissage énonciatif est opérée par la conclusion d'une anecdote qui joue sur le pathos du voyageur : elle opère ainsi une homogénéisation des deux types de récit en abolissant la distance énonciative instaurée par l'anecdote et en recentrant le discours autour du «je », par le biais de vocabulaire axiologique qui permet l'interprétation de l'anecdote par le narrateur et oriente, ce faisant, celle de l'allocutaire. Après l'anecdote sur Méhémet-Ali, le narrateur conclut :

C'est un fait qui m'a beaucoup touché. Ce pauvre vieillard qui, dans ses temps de gloire, avait été cruellement abandonné par la politique de Louis-Philippe, rêvant, malgré ses facultés défaillantes, la reconstitution d'une royauté irrémissiblement perdue, m'a toujours semblé une de ces ironies providentielles dont le bon Dieu nous fait des leçons que nous nous obstinons à ne jamais comprendre. (p. 10)

Le vocabulaire affectif permet d'énoncer un jugement sur cette histoire particulière qui a été le pivot d'une anecdote puis d'élargir la conclusion à la sphère de l'Humanité. L'emploi $\mathrm{du}$ «nous » réitéré assure ainsi le lissage entre les deux discours, l'anecdotique et l'universel qui est en fait le but du récit de voyage, adressé à un allocutaire identifié mais celui-ci ne fonctionne que comme relais du lecteur virtuel.

\subsection{Cohésion paradigmatique}

La particularité du récit de voyage réside dans le fait que son matériau existe indépendamment de sa mise en forme. Le voyage préexiste à son récit. Dans tout écrit qui se prévaut d'être compte rendu authentique, l'invention du narrateur est limitée de fait par le contrat de lecture proposé. De manière similaire, Maxime Du Camp n'invente pas d'anecdote; dès lors, on peut supposer qu'en tant qu'histoires autonomes, celles-ci préexistent au récit de voyage qui n'en est que le porte-voix. Toutefois, la sélection de ces anecdotes et leur insertion dans le récit final du voyageur les situe dans une nouvelle perspective. En regard les unes des autres, elles se répondent et finalement contribuent à la cohésion du texte : l'inscription dans la chaîne du discours, qui brise la cohésion syntagmatique sur différents plans, joue en faveur d'une cohésion paradigmatique. L'effet de récurrence de ce phénomène digressif contribue à lui donner une autre valeur. L'anecdote-digression se multiplie et, réitérant la déviation, elle acquiert la légitimité que finissent par avoir les erreurs répétées. On aboutit peut-être même à une inversion de 
priorité : toute la narration du voyageur ne servirait que de ciment entre tous ces fragments, entre toutes ces historiettes rapportées et qui deviennent paradoxalement gage de référentialité. Le discours du narrateur ne serait que discours autour des anecdotes.

Sur le plan formel, certaines constantes thématiques, énonciatives ou syntaxiques relient les anecdotes entre elles : cette chaîne qui se construit par-delà le texte-cadre contribue même à la cohésion syntagmatique du récit de voyage. Chaque anecdote brise apparemment la linéarité du texte mais elle est aussi annonce de l'anecdote suivante ; la brèche ainsi ouverte est toujours en attente d'une nouvelle brèche similaire qui la referme en la remplaçant. Ce parcours d'une ouverture à l'autre est accompli par le lecteur, seule instance qui peut percevoir la cohérence du récit de voyage.

\section{L'anecdote comme facteur de cohérence}

\subsection{Cohérence pragmatique}

L'allocutaire est, pour cette œuvre, identifié puisqu'il s'agit de Théophile Gautier. Maxime Du Camp en fait à la fois l'initiateur et le dédicataire de son récit de manière explicite, en citant ses encouragements notamment dans la Préface :

Essaye de faire partager aux autres les jouissances que tu as éprouvées ; raconte ce que tu as vu, d'autres en tireront les conséquences devant lesquelles tu sembles reculer; chante ta chanson, quelle qu'elle soit, une voix se trouvera peut-être pour qui elle aura été écrite. (Préface, Chaville, 6 juin 1853)

Tout au long de ses lettres de voyage écrites cependant après le retour en France et à partir des notes de voyage, les marques d'allocution sont récurrentes. Cet allocutaire sert de balise ; il est l'élément fixe vers lequel converge le faisceau constitué par les multiples fragments du discours du voyageur. Il est en fait une image du lecteur virtuel, le représentant d'une communauté homogène, celle à laquelle appartient le narrateur même ${ }^{11}$. La figure du lecteur qui se dessine constitue donc une entité homogène qui sert de point de repère au discours du voyageur. C'est le parcours de la lecture cursive ou récursive qui finalement donne son unité à l'œuvre et sa cohérence. L'attitude du lecteur face à des digressions peut être ambivalente : soit il gomme les disparités éventuelles,

${ }^{11}$ Le récit de voyage repose sur une trilogie, le narrateur français qui s'adresse à une collectivité, second pilier du triptyque, susceptible de comprendre son discours avec ses implicites et ses présupposés éventuels donc qui doit appartenir à la même sphère socio-culturelle que lui, et enfin le délocuté, l'Autre, en l'occurrence l'Arabe, celui dont on parle et à qui on ne prête que rarement la parole. 
élude les excursus et reconstitue la linéarité du voyage et de son récit, soit au contraire il est sensible aux retours d'éléments similaires, au rythme institué par les anecdotes digressives qu'il inscrit dans une nouvelle chaîne signifiante, en considérant le récit-cadre comme subsidiaire, ancillaire de l'anecdote. De fait, tout le discours de Maxime Du Camp est orienté par un allocutaire réel et, au-delà, par une image préconçue du lecteur virtuel. L'interaction entre celui qui écrit et celui qui lit influe sur la forme du récit de voyage. L'anecdote digressive, dans cette stratégie énonciative dialogique, a un enjeu pragmatique évident.

L'ethos ${ }^{12}$ du narrateur-voyageur transparaît dans son discours : il est celui qui a vu et qui transcrit avec la plus grande transparence possible : c'est en tout cas le pacte de lecture inaugural du récit de voyage en général et du Nil en particulier; Du Camp entreprend d'écrire «ce qu'(il) a vu dans les pays lumineux qu'(il) a parcourus », toujours en quête «des vérités éparses parmi les peuples qui couvrent la terre ${ }^{13}$. La mission que se donne le voyageur dans son récit est celle du témoin. Dès lors, il se trouve partagé entre deux tendances du voyageur, divisé entre deux types de savoirs, la doxa et l'epistemé, pour reprendre des notions de la rhétorique antique. Il est celui qui sait, que la source de son savoir soit culturelle ou expérimentale. Les deux types d'anecdotes se distribuent ainsi selon qu'elles sont merveilleuses ou historiques. Le narrateur est l'écho de tout ce qu'il entend ou voit. Il est investi du savoir et dispose du pouvoir de la parole, mais il est aussi astreint au devoir de dire comme en témoigne cette formule à la modalité déontique qui prétend satisfaire à la maxime conversationnelle de quantité - consistant à fournir l'information pertinente maximale :

Il y a encore mille historiettes aussi sérieuses que les précédentes et dont je te fais grâce ; cependant, il en est une que je ne dois pas oublier. (p. 74)

Mais cette maxime est elle-même dominée par l'axiome de pertinence que l'anecdote, par sa nature digressive, enfreint a priori. Néanmoins, si le discours est pourvoyeur d'un savoir nouveau - conformément à l'étymologie qui désigne sous ce nom une chose non publiée - il se trouve alors légitimé. C'est ainsi que les anecdotes trouvent leur raison d'être et contribuent à la cohérence de l'œuvre. Maxime Du Camp insiste sur le caractère inédit de l'une d'entre elles :

\footnotetext{
12 L'ethos peut être défini comme l'image que l'orateur projette de lui-même dans son discours et qui contribue à sa crédibilité et autorité. Voir le rappel de Amossy 2000.

13 Préface.
} 
Puisque je te parle de Méhémet -Ali, dont, entre nous, on a trop exagéré la valeur, je te raconterai sur lui une courte anecdote fort caractéristique et peu connue.

Trois arguments sont avancés comme pour prévenir l'objection éventuelle de hors-sujet : la brièveté, la représentativité et la nouveauté de l'anecdote.

Le voyageur est un témoin qui doit rendre compte de ce qu'il a vu ou entendu pour une leçon de choses magistrale. Cependant, il n'écrit pas un ouvrage strictement scientifique ; d'ailleurs, placer le récit sous l'égide de Théophile Gautier paraît d'emblée lui conférer une tonalité plus littéraire. Le récit de voyage se distingue de l'ouvrage savant par la veine romanesque qui le traverse et qui se trouve concrétisée par la composition même des anecdotes digressives.

Incluses dans le récit de voyage, elles constituent en effet des micro-histoires qui respectent une dynamique narrative. L'alternance entre passés simples et imparfaits, spécifiques des textes narratifs, de même que la présence d'adverbes connecteurs temporels caractérisent plusieurs anecdotes ; par exemple celle qui relate la fécondation inespérée du palmier femelle au jardin des Plantes à Paris par un palmier de Marseille ou encore l'histoire de l'anachorète et des crocodiles :

Autrefois il y avait à Paris, au jardin des plantes, un palmier femelle ; il était seul de son espèce et mourait de langueur; chaque année, quand arrivait le printemps, il espérait des baisers balsamiques, mais ceux-ci ne venaient jamais, et le pauvre arbre se consumait dans les désirs de la stérilité.

Enfin, une fois, il conçut et porta des fruits. Les savants ébahis mirent leurs lunettes et vinrent voir ce prodige.

Il n'y avait pas à en douter, un régime de jeunes dattes pendait sous les feuilles. On fit des calculs alors, on rechercha où pouvait se trouver le palmier générateur qui avait enfin eu pitié de cette longue chasteté involontaire. Le palmier mâle le plus voisin se balançait dans le jardin botanique de Marseille.

Deux cents lieues à vol d'oiseau, un grain de pollen les avait parcourues sur l'aile du vent pour que la loi même de la nature - qui est la reproduction - ne fût pas plus longtemps outragée. (p. 1834)

Un saint homme vivait autrefois au milieu des ruines d'Antinoë. La vieillesse avait brisé sa vigueur, affaibli sa vue et perclu ses membres; à peine trouvait-il encore la force de faire ses ablutions.

Quant à ses prières, il les récitait mentalement tout le jour. 
Il n'avait d'autre serviteur qu'un âne qui chaque jour allait au fleuve puiser l'eau nécessaire aux besoins de son maître ; il descendait au rivage, portant dans sa bouche deux vases suspendus à un bâton, les emplissait l'un après l'autre et revenait à la hutte qu'habitait le pieux cénobite.

Depuis deux ans jamais il n'avait failli à sa tâche. Un jour de grande chaleur et qu'il était fort tourmenté par les mouches, il regarda le fleuve avec mélancolie et pensa qu'il jouirait d'une bonne fraîcheur s'il pouvait se plonger dans les ondes murmurantes ; le diable souffla quelques mauvais conseils dans ses longues oreilles; le pauvre innocent bourriquet ne sut pas résister aux tentations de l'esprit malin ; il déposa sur le bord ses vases encore vides et descendit dans le Nil en brayant de plaisir. Un crocodile passait par là, il happa le baigneur et n'en fit que deux bouchées.

Cependant l'anachorète attendait le retour de son âne ; il attendit le soir, il attendit toute la nuit ; alors l'inquiétude serra son cœur ; il fit un grand effort, se traîna jusqu'aux berges du Nil, et y trouvant les vases intacts, ne douta plus que son serviteur fidèle n'eût été la proie d'un crocodile ; il leva les mains vers le ciel, pria Dieu, invoqua le prophète, maudit les crocodiles et rentra dans sa demeure en pleurant.

La malédiction a été féconde, car, depuis, jamais un crocodile n'a pu franchir le Nil au delà de Cheikh-Abadeh. (p. 107-108)

La présence de l'allocutaire est manifeste au travers de toutes les marques attendues d'allocution mais il peut aussi se dessiner en creux au travers d'opinions que le narrateur lui prête ou encore au travers d'implicites qui fondent les stéréotypes. L'incomplétude des énoncés n'est acceptable que si l'allocutaire est supposé capable de pallier les lacunes. Les anecdotes sont choisies en fonction de deux motifs élus constantes a priori du peuple arabe décrit : la violence et le merveilleux. L'énonciateur s'inscrit dans une topique qu'il estime partagée par le groupe homogène qu'il construit comme lecteur de son œuvre.

Je comprends que les Arabes, toujours cherchant le merveilleux, aient peuplé ces solitudes avec les génies dont Eblis est le chef et que Soliman Ben Daoud avait enchainés sous son trône.

Ces différents facteurs qui impliquent la réception et son univers d'attente font envisager le texte à un autre niveau, celui de son appartenance générique.

\subsection{Au niveau générique}

De même que la récurrence de séquences anecdotiques construites sur le même modèle contribue à la cohésion syntagmatique et paradigmatique du récit de voyage, l'anecdote envisagée comme motif itératif d'un certain type de récit peut être érigée en constante générique. Est-on alors dans l'ordre de la cohésion ou de la cohérence du texte ? Si on 
envisage la relation intertextuelle entre les différents récits de voyage dans une perspective diachronique, sans doute est-on dans le domaine de la cohésion ; si d'un point de vue synchronique, on suppose une relation d'inclusion d'un récit de voyage particulier par rapport à une norme, la relation architextuelle nous place du côté de la cohérence. On peut alors suivre Jean-Marie Schaeffer pour qui l'architexte n'est pas un ensemble de textes particuliers mais un modèle de lecture construit par induction à partir d'un ensemble d'hypotextes (J.-M. Schaeffer, p. 201), une sorte de texte idéal ou une matrice, construite sur des récurrences ou stéréotypes. Le genre serait un intermédiaire entre les œuvres et la littérature. La matrice du récit de voyage pourrait être l'anecdote qui permet la traversée d'un espace-temps autre, la traversée d'autres discours, la traversée des genres et définit un modèle générique, celui du récit de voyage.

\section{Conclusion}

L'anecdote digressive est, par nature, un élément d'hétérogénéité dans la trame linéaire du récit de voyage : elle introduit une rupture sur le plan temporel et énonciatif puisqu'elle se constitue comme un fragment autonome, clos sur lui-même et répondant à ses propres critères de régulation. Elle est un facteur de rupture de la cohésion du récit-cadre et menace de fait sa cohérence, puisqu'elle intervient comme fragment parasite. Le récitcadre a pour rôle d'en délimiter les contours, de dessiner les frontières comme pour maîtriser la dangereuse hétérogénéité. Il essaie de l'intégrer en exhibant son rôle ancillaire par exemple en l'embrigadant dans une relation logique de causalité, ou en l'enserrant entre des bornes. Le discours du narrateur tend à lisser les aspérités temporelles et énonciatives pour rétablir le suivi de son discours assuré par des marques cohésives explicites. A ce niveau, cohésion et cohérence semblent solidaires : que celle-ci soit brisée et c'est la cohérence qui est menacée ; qu'elle s'exhibe au contraire - quoique de manière rhétorique et artificielle - et la cohérence semble rétablie. Toutefois, la cohérence fondamentale du récit de voyage se passe de marqueurs explicites de cohésion et repose paradoxalement sur l'anecdote même. Un traitement cumulatif, l'enfilade des anecdotes, contribue à la cohésion syntagmatique et paradigmatique ; mieux encore, c'est du côté de la réception qui fait envisager la perspective générique que le rôle de l'anecdote est le plus évident.

L'anecdote digressive est la matrice du récit de voyage à la fois dans sa structure formelle et dans sa dialectique. Le premier aspect pourrait se résumer dans l'opposition de l'ouvert et du fermé : le récit de voyage est souvent défini comme un genre à la croisée d'autres genres. L'anecdote, élément clos sur lui-même, contribue à ouvrir constamment le texte- 
cadre pour finalement lui donner sa cohérence, donc le refermer. Les ruptures isotopiques, les variations sont un des principes du genre pleinement illustrés par le parangon de l'anecdote digressive, sorte de voyage dans le voyage. D'autre part, quel motif plus représentatif que l'anecdote digressive peut-on trouver comme illustration du mécanisme du récit de voyage ? Le voyageur est confronté au différent, à l'hétérogène au cours de son périple, qu'il s'efforce de réduire pour le comprendre ; son récit, de manière similaire, est parsemé des éléments insolites que sont les anecdotes digressives, comme une représentation verbale du choc de deux discours ou de deux univers. Tout l'effort du narrateur est de lisser les différences pour rendre son discours cohérent, comme le mouvement du voyageur est de réconcilier le même et l'autre pour s'approprier l'univers qu'il perçoit.

\section{Bibliographie}

Du Camp Maxime, [1854], 1877, Le Nil, Egypte et Nubie, Paris, Hachette.

\section{Ouvrages critiques}

Amossy Ruth (2000). L'Argumentation dans le discours, Paris, Nathan.

Dupriez Bernard (1984). Gradus, Les Procédés littéraires, Paris, Union Générale d'Editions (10/18).

Jaubert Anna (1993). «A propos, que je te dise... et autres pertinentiseurs. Un rapport entre les modalisateurs d'énonciation et les connecteurs propositionnels » in Actes du XXe Congrès international de Linguistique et Philologie Romanes, T. I, Tübingen, Francke Verlag, p.317-328.

Magri Véronique (1995). Le Discours sur l'Autre, Paris, Champion.

Maingueneau Dominique (1993). Le Contexte de l'œuvre littéraire, Enonciation, écrivain, société, Paris, Dunod, 1993.

Morier Henri (1998). $5^{\mathrm{e}}$ édition, Dictionnaire de poétique et de rhétorique, Paris, PUF (coll. Grands dictionnaires).

Schaeffer Jean-Marie (1989). Qu'est-ce qu’un genre littéraire ?, Paris, Seuil (Poétique). 
Le statut de l'anecdote digressive 